\title{
Sensations induced by medium and long chain triglycerides: role of gastric tone and hormones
}

\author{
R Barbera, M Peracchi, F Brighenti, B Cesana, P A Bianchi, G Basilisco
}

\begin{abstract}
Background-The relative roles of gastric relaxation and the neuroendocrine signals released by the small intestine in the perception of nutrient induced sensations are controversial. The different effects of long chain (LCT) and medium chain (MCT) triglyceride ingestion on perception, gastric relaxation, and hormonal release may help to elucidate the mechanisms underlying nutrient induced sensations. Aims-To compare the effects of intraduodenal LCT and MCT infusions on perception, gastric tone, and plasma gut hormone levels in healthy subjects.

Subjects-Nine fasting healthy volunteers. Methods-The subjects received duodenal infusions of saline followed by LCTs and MCTs in a randomised order on two different days. The sensations were rated on a visual analogue scale. Gastric tone was measured using a barostat, and plasma gut hormone levels by radioimmunoassay.

Results-LCT infusion increased satiation scores, reduced gastric tone, and increased the levels of plasma cholecystokinin, gastric inhibitory polypeptide, neurotensin, and pancreatic polypeptide. MCT infusion reduced gastric tone but did not significantly affect perception or plasma gut hormone levels. LCTs produced greater gastric relaxation than MCTs.

Conclusions-The satiation induced by intraduodenal LCT infusion seems to involve changes in gastric tone and plasma gut hormone levels. The gastric relaxation induced by MCT infusion, together with the absence of any significant change in satiation scores and plasma hormone levels, suggests that, at least up to a certain level, gastric relaxation is not sufficient to induce satiation and that nutrient induced gastric relaxation may occur through cholecystokinin independent mechanisms. (Gut 2000;46:32-36)
\end{abstract}

Keywords: gastric tone; triglyceride; hormones; satiation; cholecystokinin; nutrients

The relative roles of gastric relaxation and the neuroendocrine signals released by the small intestine in the perception of the sensations induced by nutrients are still unclear. Changes in the chemical composition of nutrients infused into the duodenum lead to different sensations despite a similar increase in gastric distensibility, which suggests that the neuroendocrine signals released by the small intestine influence nutrient induced sensations. ${ }^{12}$ Of the various hormones released by the interaction of nutrients with the small intestine, cholecystokinin (CCK) has been shown to play a crucial role in inducing the sensations associated with long chain triglyceride (LCT) ingestion. ${ }^{34}$ On the other hand, studies performed using an electronic barostat have shown that changes in gastric tone modulate perception in healthy subjects, ${ }^{5}$ and that impaired gastric relaxation may be involved in the pathogenesis of dyspepsia. ${ }^{67}$

The effects of LCTs on perception, gastrointestinal motility, and plasma hormone levels differ considerably from those of medium chain triglycerides (MCTs). LCTs induce satiation, fullness and nausea, ${ }^{1-4}$ decrease gastric tone, ${ }^{8}$ and increase plasma gastric inhibitory peptide, pancreatic polypeptide, and CCK levels. ${ }^{9}$ MCTs reduce food intake, but this effect does not seem to involve the perception of satiation or fullness ${ }^{10}$; furthermore, they only weakly stimulate plasma CCK, and have no effect on gastric inhibitory peptide and pancreatic polypeptide levels. ${ }^{9}$ The effect of MCTs on gastric tone is still not known, but intragastric medium chain fatty acids (10 carbon long chain) have been found to relax the gastric fundus regardless of any concomitant CCK release. ${ }^{11}$ If intraduodenal MCTs relax the gastric fundus, this would provide an example of gastric relaxation unrelated to significant changes in plasma gut hormone levels and perception, and would support the hypothesis that gastric relaxation per se may occur without any major change in perception.

The aim of our study was to compare the different effects of LCT and MCT duodenal infusions on perception, gastric tone, and the plasma levels of gut hormones in an attempt to identify the relative roles of gastric relaxation and the neuroendocrine signals released by the small intestine in the perception of nutrient induced sensations.

\section{Materials and methods}

SUBJECTS

The experiments were performed in nine healthy subjects (three women and six men aged 20-56 years), none of whom complained of dyspepsia, had a past history of any gastrointestinal disease or surgery, or was receiving any medication at the time of the study. The study protocol was previously approved by the ethics committee of Milan's Ospedale Maggiore, and all of the subjects gave their written informed consent to participate.

Abbreviations used in this study: LCT, long chain triglyceride; MCT, medium chain triglyceride; CCK, cholecystokinin.
Accepted for publication 18 August 1999 
RECORDING SYSTEM

The electronic barostat ${ }^{8}$ used to record gastric tone consists of a pressure transducer linked through an electronic feedback mechanism to an air injection-aspiration system driven by a computer (Synectics Visceral Stimulator; Medtronic Synectics Medical, Milan, Italy). The air flow rate was $38 \mathrm{ml} / \mathrm{s}$. The barostat's internal compliance was $0.5 \mathrm{ml} / \mathrm{mm} \mathrm{Hg}$, and was linear in the range of pressures from 0 to $55 \mathrm{~mm} \mathrm{Hg}$. Air compressibility was taken into account by calculating volume as: volume without correction $(0.5 \mathrm{ml} / \mathrm{mm} \mathrm{Hg} \times$ measured pressure). The pressure transducer and the air injectionaspiration system were independently connected by a double lumen polyvinyl tube (Salem Sump Tube; Sherwood Medical, Petit Rechain, Belgium; outer diameter $4.7 \mathrm{~mm}$ ) to a highly compliant intragastric bag (Mobile Chemical Company, Pittsford, New York, USA) with a maximum capacity of 1 litre. To measure gastric tone, the barostat was set at a constant pressure: under these conditions, sustained volume expansion reflects a tonic relaxation of the stomach, and vice versa. The intrabag pressure and volume were recorded by a computer at a sampling rate of $16 \mathrm{~Hz}$ using version 2.02 of the Polygram for Windows program (Medtronic Synectics Medical). The barostat was equipped with a safety system that blocked any procedure if the intrabag pressure exceeded $55 \mathrm{~mm} \mathrm{Hg}$ for five seconds.

A single lumen polyvinyl tube (outer diameter $2 \mathrm{~mm}$, length $109 \mathrm{~cm}$; Merck Ltd, Alton, UK), equipped with a side opening located 5 $\mathrm{cm}$ from its tip, was used to perfuse saline solution and LCT and MCT emulsions into the duodenum. Duodenal motility was recorded using two manometric catheters (Portex Limited, Hythe, Kent, UK; inner diameter 0.63 $\mathrm{mm}$, outer diameter $1.4 \mathrm{~mm}$ ), tightly attached to the duodenal infusion tube, and respectively positioned 1 and $5 \mathrm{~cm}$ above the infusion port. These catheters were perfused with water through a low compliance pneumohydraulic perfusion pump (Arndorfer Specialities Inc, Greendale, Wisconsin, USA) at a flow rate of $0.5 \mathrm{ml} / \mathrm{min}$. The individual catheters were connected to external pressure transducers (Sensormedics, Anaheim, California, USA), and the pressures were transmitted from the transducers to a computer using a PC Polygraph (Medtronic Synectics Medical). The intraduodenal pressure patterns were used to monitor the position of the infusion port within the proximal part of the duodenum and the timing of the duodenal infusions.

INFUSIONS

Three preparations were tested: $0.9 \%$ saline (300 mOsm) and 20\% LCT and 22\% MCT emulsions. The two emulsions contained the same amount of fatty acids (18\%) and lecithin $(1.5 \%)$. The fatty acids were mainly oleic $(26 \%)$ and linoleic $(52 \%)$ in the LCT emulsion and octanoic $(56 \%)$ and decanoic $(40 \%)$ in the MCT emulsion. The total glycerol content $(4.5 \%)$ was equalised by adding respectively 2.5 and $0.95 \%$ glycerol to the LCT and MCT emulsions. The two emulsions had the same energy content and osmolarity ( $350 \mathrm{mOsm})$. All three preparations were administered intraduodenally using a syringe pump (Braun, Melsungen, Germany) at a constant rate of 1 $\mathrm{ml} / \mathrm{min}$, corresponding to an energy delivery of $8.36 \mathrm{~kJ} / \mathrm{min}$ for the lipid infusions. The subjects were unaware of the nature of the infusions as the tubing was opaque and the pump was behind the bed.

\section{PROCEDURE}

The subjects were studied on two occasions during which their sensory, motor, and endocrine responses were evaluated while the duodenum was perfused with saline and then LCT or MCT emulsion. The order of the studies was randomised, and they were separated by a time interval of at least one week. After an overnight fast, the probe used for the duodenal infusions was introduced through the nose on the morning of the study, and its position checked by fluoroscopy; the infusion port was close to the ligament of Treitz about $20 \mathrm{~cm}$ distal to the pylorus. Once the duodenal tube was in position, the subjects swallowed the tube of the barostat with the tied bag tightly wrapped around its end. The bag was positioned in the fundus of the stomach and then gradually inflated using less than $600 \mathrm{ml}$ of air; it was then completely deflated and connected to the barostat. The experiment started with the subject in a recumbent position at $30^{\circ}$. The individual recording pressure was determined by stepwise inflations of the bag in $1 \mathrm{~mm} \mathrm{Hg}$ increments. The pressure level that first detected respiratory excursions was considered to be the minimum gastric distending pressure, and the gastric operating pressure was then set $2 \mathrm{~mm} \mathrm{Hg}$ above this level. The mean operating pressure was $8 \mathrm{~mm} \mathrm{Hg}$ (range 6-11), and was not perceived by the subjects.

Each experiment consisted of two consecutive 30 minute duodenal infusions, the first of isotonic saline, and the second of LCTs or MCTs, both performed during a phase of quiescence (phase I) or intermittent activity (phase II) of the interdigestive migrating motor complex. At the end of each infusion period, the subjects were asked to rate the level of gastric sensations using a questionnaire, and a blood sample for gastrointestinal hormone determinations was withdrawn through a forearm venous cannula. The subjects tolerated the tubes well. In two patients, the LCT infusion was stopped after 21 and 23 minutes because of unbearable nausea; the sensation scores were recorded and the blood samples taken at these times.

SENSATION SCORES

The subjects had no previous experience of comparable studies of visceral sensitivity, and all received standard instructions. The following sensations were measured: satiation (a feeling of repletion, similar to that induced by meal ingestion, that stops further food ingestion), fullness (a feeling of complete satiation, so intense to be uncomfortable), abdominal bloating (a feeling of uncomfortable abdominal distention), nausea (a feeling of sickness with distaste for food and an urge to vomit), and pain. Each sensation was 
scored independently on a visual analogue scale consisting of a $100 \mathrm{~mm}$ line, with 0 representing "no sensation" and $100 \mathrm{~mm}$ the "strongest sensation ever felt".

\section{GASTROINTESTINAL HORMONES}

The blood samples were collected in ice chilled tubes containing EDTA $(1 \mathrm{mg} / \mathrm{ml})$ and aprotinin $(500 \mathrm{KIU} / \mathrm{ml})$. The plasma was separated by centrifugation at $4^{\circ} \mathrm{C}$ and stored in aliquots at $-80^{\circ} \mathrm{C}$ until assayed. Gastrointestinal peptides were measured by radioimmunoassay in plasma extracted on SepPak $\mathrm{C}_{18}$ cartridges. $^{12}$ Commercially available kits (Peninsula Laboratories, Inc, Belmont, California, USA) were used for the somatostatin, pancreatic polypeptide, neurotensin, and gastric inhibitory peptide assays. CCK was measured as previously described $^{13}$ using a commercially available antibody (C2581; Sigma Chemical Co, St Louis, Missouri, USA) raised in rabbits against synthetic sulphated CCK-8. The 95\% confidence limits of detection were respectively 1.5, $1.4,0.95,8.0$, and $1.2 \mathrm{pg} /$ tube, and the intraand inter-assay coefficients of variation were respectively $7.1,9.5,4.4,7.2$, and $6.4 \%$ and $5.2,9.6,5.9,9.8$, and $11.7 \%$.

DATA HANDLING AND STATISTICAL ANALYSIS

The mean gastric volume during the last five minutes of each infusion was used to measure gastric tone. The effects of LCTs and MCTs were compared after correction for the effects of the saline infusion. Analysis of variance for a cross over design ${ }^{14}$ was performed on the differences between the effects of LCTs and MCTs and those of the respective saline infusions considered as the baseline. The

Table 1 Sensation scores ( $m$ m on a visual analogue scale with $0 \mathrm{~mm}$ representing "no sensation" and $100 \mathrm{~mm}$ "strongest sensation ever felt") after intraduodenal infusions of saline and long and medium chain triglycerides (LCT and $M C T$ )

\begin{tabular}{llllll}
\hline & Saline & LCT & Saline & MCT & p Value \\
\hline Satiation & $13(15)$ & $69(37)$ & $8(18)$ & $20(35)$ & $0.006^{\star}$ \\
Nausea & $3(7)$ & $43(41)$ & $6(10)$ & $13(21)$ & 0.024 \\
Bloating & $27(24)$ & $32(30)$ & $16(15)$ & $22(14)$ & 0.583 \\
Fullness & $28(25)$ & $37(32)$ & $15(12)$ & $21(19)$ & 0.874 \\
Pain & $2(6)$ & $3(10)$ & $4(2)$ & $2(6)$ & 0.045 \\
\hline
\end{tabular}

Data are mean $(\mathrm{SD})$.

${ }^{\star}$ Considered as statistically significant as it is lower than the statistically significant threshold ( $\mathrm{p}=0.01)$ according to Bonferroni's correction for multiple comparisons. Moreover, satiation scores after LCT infusion were significantly greater than after saline infusion ( $\mathrm{p}=0.001)$; all of the other comparisons between the triglycerides and their related saline infusions were not statistically significant.

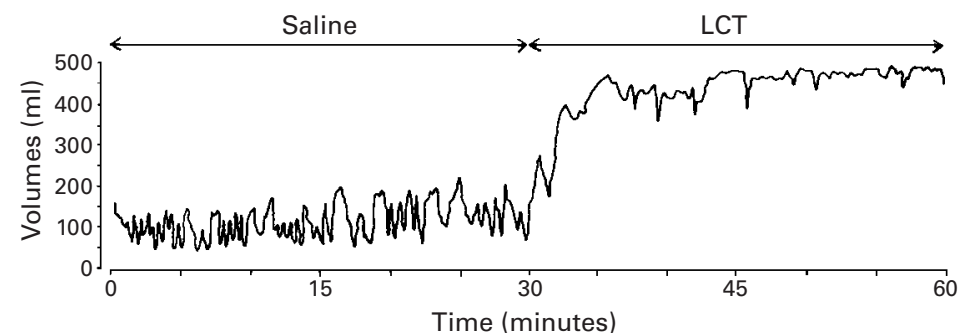

Figure 1 Changes in gastric tone during intraduodenal saline and long chain triglyceride (LCT) infusion in one subject. Note the immediate and sustained gastric relaxation shown by the increase in gastric volume of the barostat bag in response to the lipid infusion. The operating pressure (not shown in the figure) was $8 \mathrm{~mm} \mathrm{Hg}$ throughout the experiment. statistical analysis was performed after the logarithmic transformation of gastric volumes and plasma gastrointestinal hormone levels, and after the square root transformation of sensation scores, in order to achieve a Gaussian distribution of the investigated variables. For the sake of clarity, the data are reported as mean (SD) of the non-transformed data. To improve the confidence level of our conclusions, Bonferroni's correction for multiple comparisons was applied within each group of variables (visual analogue scale, gastric tone, and hormone measures).

\section{Results}

SENSATION SCORES

Table 1 shows the sensation scores after the intraduodenal infusions of saline, LCTs, and MCTs. The 56 (33) $\mathrm{mm}$ increase in satiation score after LCT infusion was significantly different from the 12 (18) $\mathrm{mm}$ increase after MCT infusion ( $p=0.006)$; furthermore, the satiation scores after LCT infusion were also significantly greater than after saline infusion $(\mathrm{p}=0.001)$. The changes in nausea and pain after LCT and MCT infusions (40 (40) $\mathrm{mm} v$ 7 (12) $\mathrm{mm}$ and 1 (23) $\mathrm{mm} \mathrm{v-2} \mathrm{(26)} \mathrm{mm}$ respectively) were not considered statistically significant according to the conservative approach chosen; bloating and fullness after the two triglyceride infusions were not significantly different. With the exception of satiation after LCT infusion, none of the comparisons between the triglyceride infusions and their saline counterparts was statistically significant.

\section{GASTRIC TONE}

Figure 1 is a representative tracing of the changes in gastric volume during the intraduodenal infusions of saline and LCTs. Gastric volume was unaffected by the former, but the latter induced a rapid and sustained relaxation of the stomach. Gastric volumes after the intraduodenal infusions of saline and LCTs were respectively 144 (35) $\mathrm{ml}$ and 480 (216) $\mathrm{ml}$, and after the infusions of saline and MCTs 156 (42) $\mathrm{ml}$ and 264 (71) $\mathrm{ml}$. The increase in gastric volume after LCT infusion (336 (193) $\mathrm{ml}$ ) was significantly greater than that observed after MCT infusion $(108(52) \mathrm{ml})(\mathrm{p}=0.001)$. The relaxation after both LCT and MCT infusions was significantly greater than after saline infusion ( $p=0.0001$ and 0.0002 respectively).

Figure 2 shows the pattern of the changes in satiation scores and gastric volumes in each subject after MCT and LCT infusions. After MCT infusion, the satiation scores increased by $20-50 \mathrm{~mm}$ in three subjects and did not change in the others; the concurrent gastric volumes increased by between 25 and $200 \mathrm{ml}$. After LCT infusion, the satiation score increased by more than $30 \mathrm{~mm}$ in all but one subject and gastric volume by more than 200 $\mathrm{ml}$ in all but two.

GASTROINTESTINAL HORMONES

Figure 3 shows the changes in plasma hormones during LCT and MCT infusions over the respective saline infusion. The changes in plasma CCK, gastric inhibitory peptide, 




Figure 2 Increases in satiation scores and gastric volumes after intraduodenal infusions of medium and long chain triglycerides (MCT and LCT) over the respective saline infusions in each subject. Each line represents one subject.

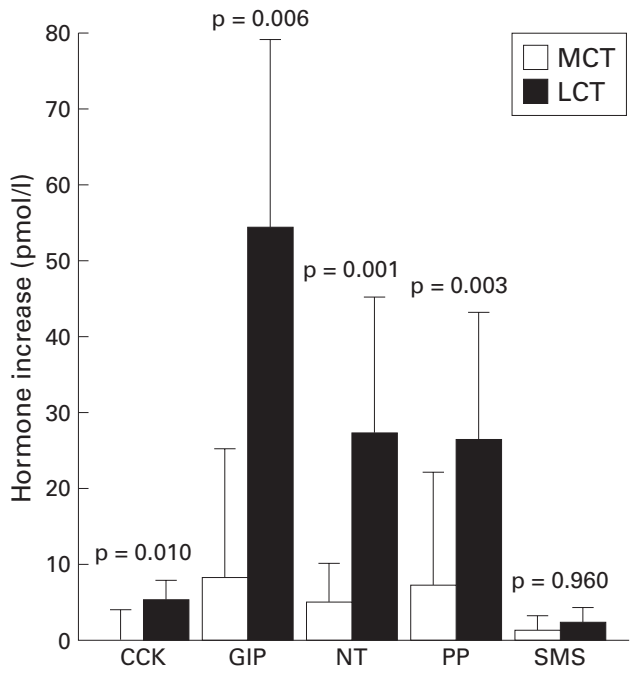

Figure 3 Increases in plasma hormone levels after intraduodenal infusions of medium and long chain triglycerides (MCT and LCT) over the respective saline infusions. Data are mean (SD). The probabilities of the differences between MCT and LCT infusions are reported. CCK, cholecystokinin; GIP, gastric inhibitory peptide; NT, neurotensin; PP, pancreatic polypeptide; SMS, somatostatin.

neurotensin, and pancreatic polypeptide, but not those in plasma somatostatin, were significantly different after LCT infusion from those after MCT infusion ( $\mathrm{p}=0.010,0.006,0.001$, 0.003 , and 0.960 respectively; fig 3). Plasma CCK, gastric inhibitory peptide, neurotensin, and pancreatic polypeptide levels were significantly greater after LCT than after saline infusion $(\mathrm{p}=0.0100,0.0001,0.0008$, and 0.0001 respectively); on the other hand, plasma levels of CCK, gastric inhibitory peptide, neurotensin, and pancreatic polypeptide after MCT infusion were not significantly different from those observed after saline infusion. Plasma somatostatin levels after both LCT and MCT infusion were similar to those recorded after saline infusion.

Figure 4 shows the pattern of the changes in satiation scores and plasma CCK levels in each subject after MCT and LCT infusions. After MCT infusion, the satiation scores increased in

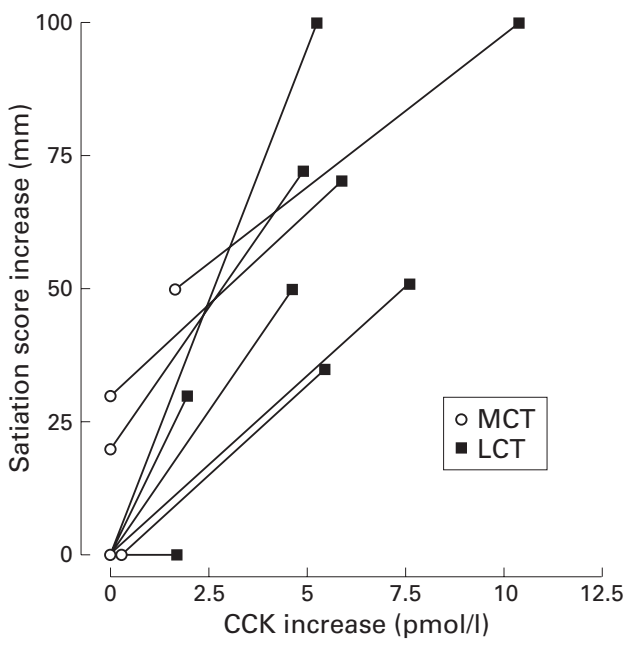

Figure 4 Increases in satiation scores and plasma cholecystokinin (CCK) levels after intraduodenal infusions of medium and long chain triglycerides (MCT and LCT) over the respective saline infusions in each subject. Each line represents one subject.

three subjects and did not change in the others, without any concurrent change in plasma CCK levels. After LCT infusion, the satiation scores increased in all but one subject, and plasma CCK levels in all but two.

\section{Discussion}

This study confirms that an intraduodenal LCT infusion induces satiation, relaxes the gastric fundus, and releases various hormones including CCK, in line with the results of previous experiments. ${ }^{1-489}$ These data, together with observations of partially blocked LCT induced sensations after the administration of the CCK-A receptor antagonist loxiglumide, ${ }^{34}$ suggest that CCK plays a role in LCT induced satiation, possibly through the stimulation of peripheral vagal afferents ${ }^{15}$ or centrally in the area postrema. ${ }^{16}$ The considerable relaxation of the gastric fundus induced by LCTs, together with other LCT induced effects on gastric motor function (not assessed in our study) such as changes in antropyloric motility, ${ }^{17}$ may also play a role, as antral distention seems to correlate well with satiation. ${ }^{13} 18$ Moreover, LCTs significantly increased plasma gastric inhibitory peptide, neurotensin, and pancreatic polypeptide levels. Gastric inhibitory peptide and neurotensin have an inhibitory effect on gastric motor function ${ }^{19}{ }^{20}$; on the other hand, their effects on nutrient induced sensations are still not known and studies with selective antagonists would be advisable.

The confirmatory results obtained after intraduodenal LCT infusion support the validity of our experimental model of assessing lipid induced changes in perception, gastric tone, and hormone plasma levels. Using the same model, we also observed the relaxation of the gastric fundus after intraduodenal MCT infusion, although the degree of this relaxation was significantly smaller. Interestingly, MCT induced relaxation occurred without any concomitant increase in satiation scores or plasma gut hormone levels including CCK. What does this result tell us about the mechanisms 
underlying nutrient induced sensations? At least up to a certain point (108 (52) $\mathrm{ml})$, gastric relaxation does not seem to be sufficient to induce satiation, a conclusion that is in line with the results of previous studies in which nutrient induced sensations have been found to change according to the different composition of the meal despite a similar degree of gastric relaxation. ${ }^{12}$ It is therefore conceivable that gastric relaxation needs to be greater than that observed after MCT infusion, or be concomitant with changes in plasma gut hormones, in order to induce sensations.

Nutrient induced relaxation of the gastric fundus follows a vagal reflex pathway ${ }^{21}$ and requires the activation of intrinsic nitrergic neurones in the stomach. ${ }^{22}$ Previous studies have shown that nutrient induced gastric relaxation can be largely, but not completely, inhibited by CCK-A receptor blockade. ${ }^{23}{ }^{24}$ Our results suggest that nutrient induced gastric relaxation can also occur regardless of the release of the gut hormones we tested including $\mathrm{CCK}$, and are in agreement with those of other studies that have found that medium chain fatty acids (10 carbon long chain) can activate CCK-independent relaxation of the proximal stomach. ${ }^{11}$

Caution is required when extrapolating the conclusions obtained using our experimental model for the short term effect of intraduodenal LCT and MCT infusion on satiation (the process that brings eating to an end) to the clinical control of satiety (the inhibition of further eating). In our study the duodenum was perfused at a rate that did not exceed the estimated maximum during normal gastric emptying, ${ }^{25}{ }^{26}$ but bypassed oral sensory stimulation and the passive distention of the gastric fundus and the antrum. Furthermore, MCTs are more potent than LCTs in inhibiting the consumption of a test meal 30 minutes after a fat preload, ${ }^{10}$ and, similarly, the isoenergetic substitution of MCTs for LCTs in high fat diets limits excess food intake over a period of two weeks and allows better control of body weight. ${ }^{27}$ Finally, an increase in the dietary MCT to LCT ratio in healthy subjects leads to a proportional increase in energy expenditure and urinary noradrenaline (norepinephrine) excretion. ${ }^{28}$ It is thus likely that factors other than CCK (such as the activation of the sympathetic nervous system, or different triglyceride oxidation rates) are involved in regulating long term energy balance when fats of different chain lengths are consumed.

In conclusion, our study confirms that the satiation induced by intraduodenal LCT infusion may involve various mechanisms, such as changes in gastric tone and plasma levels of gut hormones including CCK. It also shows for the first time that MCT infusion relaxes the gastric fundus and that this occurs without any concomitant increase in satiation scores or the plasma levels of gut hormones including CCK. These results suggest that nutrient induced gastric relaxation may occur through the activation of CCK independent mechanisms and that, at least up to a certain degree, gastric relaxation does not induce satiation.

This study was supported financially by the Associazione Amici della Gastroenterologia del Padiglione Granelli and the University of Milan.

1 Barbera R, Feinle C, Read NW. Nutrient-specific modulaion of gastric mechanosensitivity in patients with funcional dyspepsia. Dig Dis Sci 1995;40:1636-41.

2 Feinle C, Grundy D, Read NW. Effects of duodenal nutrients on sensory and motor responses of the human stomach to distention. Am $\mathcal{F}$ Physiol 1997;273:G721-6.

3 Lieverse RJ, Jansen JBMJ, Masclee AAM, et al. Effect of a low dose of intraduodenal fat on satiety in humans: studies using the type A cholecystokinin receptor antagonist loxiglumide. Gut 1994;35:501-5.

4 Feinle C, D'Amato M, Read NW. Cholecystokinin-A receptors modulate gastric sensory and motor responses to gastric distention and duodenal lipid. Gastroenterology 1996; 110:1379-85.

5 Notivol R, Coffin B, Azpiroz F, et al. Gastric tone determines the sensitivity of the stomach to distention. determines the sensitivity of the

6 Coffin B, Azpiroz F, Guarner F, et al. Selective gastric Coffin B, Azpiroz F, Guarner F, et al. Selective gastric
hypersensitivity and reflex hyporeactivity in functional dyshypersensitivity and reflex hyporeactivity in fun
pepsia. Gastroenterology 1994;107:1345-51.

7 Tack J, Piessevaux H, Coulie B, et al. Role of impaired gastric accommodation to a meal in functional dyspepsia. Gastroenterology1998;115:1346-52.

8 Azpiroz F, Malagelada JR. Intestinal control of gastric tone. Am F Physiol 1985;249:G501-9.

9 Isaacs PET, Ladas S, Forgacs IC, et al. Comparison of effects of ingested medium- and long-chain triglycerides on gallbladder volume and release of cholecystokinin and other gut peptides. Dig Dis Sci 1987;32:481-6.

10 Rolls BJ, Gnizak N, Summerfelt A, et al. Food intake in dieters and nondieters after a liquid meal containing medium-chain triglycerides. Am f Clin Nutr 1988;48:6671 .

11 McLaughlin J, Lucà MG, Jones MN, et al. Fatty acid chain length determines cholecystokinin secretion and effect on human gastric motility. Gastroenterology 1999;116:46-53.

12 Peracchi M, Basilisco G, Bareggi B, et al. Plasma somatostatin levels in patients with chronic idiopathic intestinal pseudo-obstruction. Am f Gastroenterol 1997;92:1884-6.

13 Santangelo A, Peracchi M, Conte D, et al. Physical state of meal affects gastric emptying, cholecystokinin release and satiety. Br f Nutr 1998;80:521-7.

14 In: Jones B, Kenward MG, eds. Design and analysis of crossover trials. London: Chapman and Hall, 1989.

5 Blackshaw LA, Grundy D. Effects of cholecystokinin (CCK-8) on two classes of gastroduodenal vagal afferent fibre. F Auton Nerv Syst. 1990;31:191-202.

16 Van der Kooy D. Area postrema: site where cholecystokinin acts to decrease food intake. Brain Res 1984;295:345-7.

17 Andrews JM, Rayner CK, Doran S, et al. Physiological changes in blood glucose affect appetite and pyloric motility during intraduodenal lipid infusion. Am f Physiol1998; 275:G797-804.

18 Bergmann JF, Chassany O, Petit A, et al. Correlation between echographic gastric emptying and appetite: between echographic gastric emptying
influence of psyllium. Gut 1992;33:1042-3.

19 Brown JC, Dryburgh JR, Frost JL, et al. Properties and Brown JC, Dryburgh JR, Frost JL, et al. Properties and
actions of GIP. In: Bloom SR, ed. Gut hormones. New York: actions of GIP. In: Bloom SR, ed. Gut

20 Blackburn AM, Fletcher DR, Bloom SR, et al. Effect of neurotensin on gastric function in man. Lancet 1980;1: 987-9.

21 Azpiroz F, Malagelada JR. Vagally mediated gastric relaxation induced by intestinal nutrients in the dog. Am $\mathcal{F}$ Physiol 1986;251:G727-35.

22 Desai KM, Sessa WC, Vane JR. Involvement of nitric oxide in the reflex relaxation of the stomach to accommodate food or fluid. Nature 1991;35:477-9.

23 Mesquita MA, Thompson DG, Troncon LEA, et al. Effect of cholecystokinin-A receptor blockade on lipid-induced gastric relaxation in humans. Am F Physiol 1997;273:G11823 .

24 Zerbib F, Bruley de Varannes S, Scarpignato C, et al. Endogenous cholecystokinin in postprandial lower esophaEndogenous cholecystokinin in postprandial lower esophageal sphincter function and fun

25 Cortot A, Phillips SF, Malagelada JR. Parallel gastric emptying of non-hydrolyzable fat and water after a solid-liquid meal in humans. Gastroenterology 1982;82:877-81.

26 Collins PJ, Horowitz M, Cook DJ, et al. Gastric emptying in normal subjects-a reproducible technique using a single cintillation camera and computer system. Gut 1983;24: 1117-25.

27 Stubbs RJ, Harbron CG. Covert manipulation of the ratio of medium- to long-chain triglycerides in isoenergetically dense diets: effect on food intake in ad libitum feeding men. Int $\mathcal{F}$ Obes Relat Metab Disord 1996;20:435-44.

28 Dulloo AG, Fathi M, Mensi N, et al. Twenty-four-hour energy expenditure and urinary catecholamines of humans consuming low-to-moderate amounts of medium-chain triglycerides: a dose-response study in a human respiratory chamber. Eur f Clin Nutr 1996;50:152-8. 